Albericci, J. P. \& Fletcher, J. A. (1956). J. gen. Microbiol. 14, 692-697

\title{
A Modified Method for Preparing and Counting Viable Suspensions of Tubercle Bacilli
}

\author{
By J. P. ALBERICCI AND J. A. FLETCHER \\ Microbiological Research Department, Experimental Station, \\ Portor, near Salisbury, Wiltshire
}

SUMMARY : A modified method derived from that of Fenner, Martin \& Pierce (1949) is described for the preparation of homogeneous suspensions of single organisms of one strain of Mycobacterium tuberculosis at concentrations of about $10^{8}$ single organisms per $\mathrm{ml}$. A technique is also described for the accurate assessment of viable counts in a period of 14 days.

In the study of experimental respiratory tuberculosis a reliable method is required for counting viable organisms of Mycobacterium tuberculosis in the suspensions to be sprayed and in collected samples of the cloud the animals inhale. Accuracy of count in the shortest possible time is a first essential. The technique must also be sufficiently simple and safe to allow assessment of at least ten samples with many replicates in the course of any one day of experiment. Further, the technique in use for the production of the clouds of tubercle bacilli (Henderson, 1952; Druett \& May, 1952) requires a suspension with an initial concentration of the order of $10^{8}$ organisms $/ \mathrm{ml}$. The term 'organism' is here defined as a single rod-like structure which may be composed of one to about five cells of $\boldsymbol{M}$. tuberculosis. When handling suspensions consisting mainly of single organisms this concentration has the added advantage of allowing total counts to be made easily and with reasonable accuracy. In preliminary work we used the technique of Fenner et al. (1949) (together with many modifications) and applied it to a variety of strains including one (H37 Rv) used by them. Suspensions with a high percentage of single units were obtained, but rarely at a final concentration greater than $10^{4}$ and never higher than $10^{6} / \mathrm{ml}$. Further, no success followed concentration by centrifugation of these suspensions for intractable clumping invariably occurred as a result.

The present paper describes a modification of the technique of Fenner $e t$ al. (1949) which has been used continuously for over a year in these laboratories and which fulfils most of our requirements. During this time many experiments have been made with airborne clouds of Mycobacterium tuberculosis. For these tests a bovine strain was selected, partly because it had high virulence for guinea-pigs, but principally because by suitable methods of cultivation suspensions could be obtained consisting almost wholly of single organisms. This latter property was very desirable for two reasons: first, it ensured that highly homogeneous clouds of single organisms could be produced for inhalation by experimental animals; and second, it allowed the viable count technique to be put to rigorous test. 


\section{METHODS}

Organism and method of culture. The Vallée strain of Mycobacterium tuberculosis var. bovis was used. It was stored in small tubes as freeze-dried infected mouse spleen. We have used various methods for recovering and subculturing the strain, but find the following the most satisfactory. Dubos liquid Tweenalbumin medium as modified by Fenner et al. (1949) was the principal culture fluid; the amino acid constituents were added in the form of $0.05 \%(w / v)$

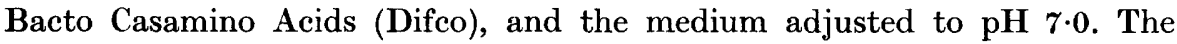
content of one tube of freeze-dried material was taken up in this medium and then inoculated on to Dorset egg medium and incubated at $37^{\circ}$ for 7 days. Subculture was then made on Dubos liquid medium and thereafter subculture was made on the same substrate at 7-day intervals. The medium was contained in $8 \times 5$ in. Roux bottles, each containing $100 \mathrm{ml}$. and a $5 \%(\mathrm{v} / \mathrm{v})$ inoculum was used. The resulting depth of liquid seemed optimal for maximum yield of viable organisms. Three to four subcultures in this medium were necessary before maximum growth of the right consistency was obtained for experimental cloud work. Thereafter, five to eight subcultures could be made without loss of virulence before reverting to another tube of freeze-dried material.

Preparations of suspensions. Immediately on removal from the incubator and before cooling to room temperature the cultures were passed through sterile folded filter-paper (Green's hyduro no. $904 \frac{1}{2}$ ). $80 \%$ of the particles in the filtrate were single units of $1 \mu^{3}$ or less. The remainder were small clumps containing two or three units each in a total viable population of $1-2 \times 10^{8}$ organisms $/ \mathrm{ml}$.

Presumptive viable count. Total counts on the suspension were immediately made in a Petroff-Hausser bacterial counting chamber, with dark-ground illumination. By comparing these total counts with those obtained by culture, accumulated evidence showed that the viable count was consistently of the order of $80 \%$ of the total count. This presumptive test finally formed a basis which allowed experiments to be made immediately with the freshly prepared suspensions.

Microvessels for colonial culture. Small lidless dishes of $35 \mathrm{~mm}$. diameter, $10 \mathrm{~mm}$. depth, with walls $2 \mathrm{~mm}$. thick, and having optically flat bases, were made for us by Messrs R. and J. Beck, Ltd., London. Each of these microvessels was contained in a 2 in. diameter Petri dish. The latter were placed in $7 \mathrm{lb}$. Kilner jars complete with glass lids and screw-retaining rings but with no rubber seal. In this manner the microvessels were hot-air sterilized and stored ready for use.

Plating medium. Dubos agar medium, as modified by Fenner et al. (1949), was prepared monthly in $100 \mathrm{ml}$. screw eap bottles. As with the liquid medium, the amino acid content was added as $0.05 \%$ (w/v) Bacto Casamino Acids (Difco); the agar used was British Drug Houses 'Japanese fine powder'. The $\mathrm{pH}$ value was adjusted to $\mathrm{pH} 6.5$ and great care was taken in filtration to ensure an optically clear gel. Immediately before use $5 \mathrm{ml}$. of $10 \%(\mathrm{w} / \mathrm{v})$ bovine 
plasma albumin (Armour Laboratories, Fraction V) in saline (pH 6.5) and $1 \mathrm{ml}$. of $50 \%(\mathrm{w} / \mathrm{v})$ citrated glucose were added to each $100 \mathrm{ml}$. of the melted agar at $56^{\circ}$. No Tween or oleic acid was incorporated in the medium because these substances markedly inhibited colony development and those colonies which appeared varied widely in size. Each microvessel was filled with $5 \mathrm{ml}$. of medium. After the agar had jelled the microvessels were incubated at $37^{\circ}$ in the inverted position in their containing Petri dishes for 18-24 hr. When not required immediately they were stored in Kilner jars at $0-4^{\circ}$. Before use the dishes, removed from the Kilner jar, were warmed in an incubator at $37^{\circ}$ for $1 \mathrm{hr}$.

Inoculation of microvessels. Dilutions of the bacterial suspension were made in $0 \cdot 1 \%(\mathrm{v} / \mathrm{v})$ bovine plasma albumin in such a manner that $0.01 \mathrm{ml}$. of the appropriate dilution was calculated from the presumptive viable count to contain 30-150 colonies. Three 0.01 ml. volumes were delivered on to the dried agar surface of each microvessel from a sterile $0 \cdot 1 \mathrm{ml}$. blood pipette controlled by a special metering device (see Appendix) which allowed high accuracy of delivery.

Incubation of the microvessels. After inoculation the microvessels were left unmoved until the deposited liquid had spread over and sunk into the agar. This occurred within a few minutes when the organisms were inoculated while still warm. The microvessels, each contained in a $2 \mathrm{in}$. Petri dish, were then incubated in the inverted position in $7 \mathrm{lb}$. Kilner jars each carrying four columns of Petri dishes, totalling 32-34/jar. A test-tube with water was placed in a centre space formed by the dishes and served to prevent desiccation of the medium. The glass lids were replaced on the Kilner jars and secured by the metal screwband only. Incubation was carried out at $37^{\circ}$. Colonies just visible to the naked eye were seen after 14 days' incubation. On removal from the incubator there was no evidence of desiccation of the agar surface or condensation of water within the jars. While the dishes were still warm, a drop (c. $0.05 \mathrm{ml}$.) of $40 \%(\mathrm{w} / \mathrm{v})$ formaldehyde was placed on the underside of the lids of the Petri dishes, which were then replaced in the jar as for incubation. It was found that after $4 \mathrm{hr}$. at $37^{\circ}$ the microvessels were sterile. Colonies removed by platinum loop and transferred to $10 \mathrm{ml}$. amounts of Dubos liquid miedium consistently failed to grow even after prolonged incubation at $37^{\circ}$; untreated colonies similarly tested consistently yielded fully grown cultures within 10 days of incubation.

Colonial counts aided by optical projection. Repeated tests showed that there was no increase in the number of colonies after 14 days. However, their size at this time prevented accurate counting by the naked eye. Counting could be done by examination under a plate microscope, but when large numbers of microvessels were in use this process quickly led to fatigue and inaccuracy. Counting was made much easier by the use of an optical projection system designed to accommodate the microvessels. The projector was arranged to throw an image of the inoculated area on a glass bead screen in a darkened room. The optically flat bases of the microvessels prevented image distortion, and large numbers of counts could thus be quickly and accurately made. 
The projector lens was a cinematograph anastigmat of focal length 2 in. and aperture f. 1·6, giving a wide flat field. The remainder of the apparatus, consisting of a force-cooled projection lamp and condenser, was of conventional design. The magnification of the image was about $\times 80$ at $14 \mathrm{ft}$. from the projector. For convenience in counting, the glass bead screen was divided by black cotton thread into 4 in. squares. By this method all colonies grown from one measured drop were illuminated in one field. The optimum number of colonies for counting was probably about 50, but reasonable accuracy could be expected in the range of 30-150 colonies. Plate 1 shows the type of picture encountered. Each colony developed a well-marked central spot. This spot was a particularly useful aid when several colonies arose close together, as seen in the upper left-hand part of Fig. 1. The extreme bottom right-hand corner of Pl. 1, fig. 1 shows, in partial illumination, colonies forming part of another measured drop. Also in the right-hand corner there is seen a well-defined 'dust' spot. This minor defect was not uncommon, but there was no difficulty in deciding which was, or was not, a colony of tubercle bacilli.

\section{RESULTS}

In eighteen consecutive experiments with clouds of single organisms, the suspension used was tested for viable count before and after the experiment, and two to ten collected cloud samples per experiment were similarly tested. The number of replicate microvessels used in any assay of suspension or cloud sample varied from three to four but there was a 2-3\% loss as a result of either extraneous contamination or the merging of drops on the agar surface in microvessels.

Viable counts of micro-organisms in equal drops taken from the same suspension may be expected to follow a Poisson distribution, but the experimental errors are such that so far as we are aware the ideal has never been achieved. In fact, it would seem from any published data, or experience in our laboratories, that $90 \%$ of samples giving adequate 'goodness of fit' (Fisher, 1946) represents a high measure of success. The data from the present experiments were examined statistically by our colleague Mr S. Peto. He fitted a Poisson distribution to each assay and examined individual drop counts for aberrant values. Among ninety-six assays there were nine such counts so small or so large as to have a probability of about $1 \%$ (=approximately $1 / 96$ ) or less, of occurring in the sample to which each belonged. These nine were rejected and new Poisson distributions were fitted to the assays affected. The value of $\chi^{2}$ was computed for every distribution. The sum of $\chi^{2}$ for assays of liquid suspensions gave a probability of $10 \%\left(\Sigma \chi^{2}=358 \cdot 33 ; n=36\right)$; for assays of cloud samples the probability was $24 \% .\left(\Sigma \chi^{2}=516 \cdot 66 ; n=60\right)$. Finally, there was only one assay (of the first cloud sample taken) that was statistically hopelessly erratic. We consider, therefore, that the soundness of the method is established beyond reasonable doubt. 


\section{DISCUSSION}

Fenner $e t$ al. (1949) raised the status of the quantitative assessment of tubercle bacilli from the position of expressing dosage in terms of fractions of a milligram or portions of a loopful to one of reasonable accuracy in terms of viable organisms. The present work is best regarded as introducing modifications to their technique, leading to possible general improvements. For our preliminary studies on airborne infection it certainly has marked advantages. The density of suspensions of single organisms that can be obtained is sufficient to make direct total counts and to produce suitable cloud concentrations to which animals can be exposed. The assay is completed in 14 days. The serious problems of either desiccation of agar or excessive condensation with concomitant contamination during incubation have been simply overcome. Economy in space and materials (particularly in plasma albumin) is, from our point of view, very important. A high degree of precision in counting has been achieved and large numbers of samples can be readily handled at short notice. However, many other strains of Mycobacterium tuberculosis would require to be successfully tested before these advantages could be accepted as being generally applicable in studies with this organism.

We are grateful to Dr D. W. Henderson for initiating this research. He has also given valuable assistance in the preparation of the paper. We wish to thank Dr C. Pierce of the Rockefeller Institute for guidance during the time she worked in our Department. We are grateful also to our colleagues Mr E. O. Powell, who designed the optical projection system, $\mathrm{Mr}$ Clement, who constructed this instrument and the pipetting device, and Mr S. Peto for the statistical analysis of results of viable counts.

\section{REFERENCES}

Druett, H. A. \& May, K. R. (1952). A wind tunnel for the study of airborne infections. J. Hyg., Camb. 50, 69.

Fenner, F., Martin, S. P. \& Pierce, C. H. (1949). The enumeration of viable tubercle bacilli in cultures and infected tissues. Ann. N.Y. Acad. Sci. 52, 751.

Fisher, R. A. (1946). Statistical Methods for Research Workers. Edinburgh: Oliver and Boyd.

Henderson, D. W. (1952). An apparatus for the study of airborne infections. J. Hyg., Camb. $50,53$.

\section{APPENDIX}

\section{Metering device, construction and use}

Fig. I shows a sketch of the instrument. It consists of a brass cylinder $2 \frac{3}{4} \times \frac{3}{4}$ in., bored for a distance of $1 \frac{1}{16}$ in. with a $\frac{11}{32}$ in. $\times 40$ t.p.i. drill and then for a further $1 \frac{1}{16}$ in with a $\frac{3}{10}$ in. drill which was unthreaded. At the other end the cylinder was bored with a $\frac{5}{8}$ in. drill for a distance of $\frac{5}{8}$ in.; this space was filled with a rubber bush for holding a pipette. A metal washer with an internal aperture smaller than the bush was first inserted in the space as a check on the 
Journal of General Microbiology, Vol. 14, No. 3

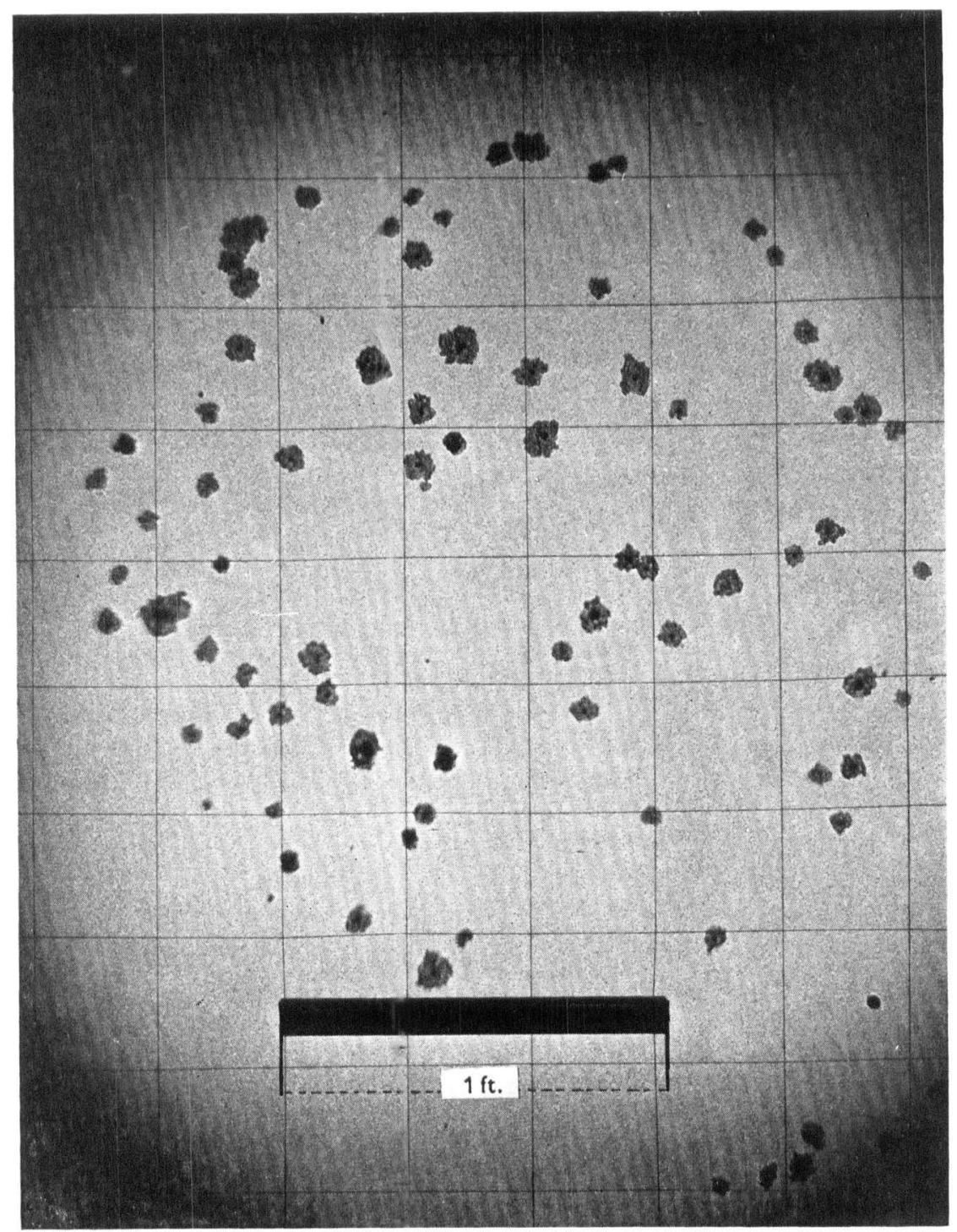

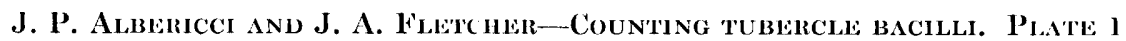

(Facing p. (697) 
depth to which the pipette could be inserted. A male counterpart for the cylinder was fitted as shown in the figure threaded at one end and made a perfect screw fit by lapping into the cylinder thread. The unthreaded extension of the spindle was made to give a sliding fit with the corresponding part of the cylinder. A 1 in. metal knob was fixed in the spindle to allow of easier rotation by digital pressure. The instrument was finished in dull nickel plate. The threaded parts and plunger were greased to give smooth action and to make an airtight seal.

In operation a sterile pipette with cottonwool plug is fitted in the rubber bush and the spindle screwed down almost to fullest extent. The pipette is charged by immersing the tip in the bacterial suspension and turning the knob of the spindle anticlockwise by thumb pressure, until the pipette contains a volume just in excess of $0.1 \mathrm{ml}$. Then, by clockwise rotation of the spindle, the excess liquid is deposited on to the surface of a spare agar plate. Three microvessels are then inoculated with three volumes of $0.01 \mathrm{ml}$, the remaining 0.01 ml. being discarded. Each $0.01 \mathrm{ml}$. is first extruded from the tip of the pipette, which is then placed on the agar and the liquid thus removed. By this means not only accurate volume delivery is secured but splashing is avoided.

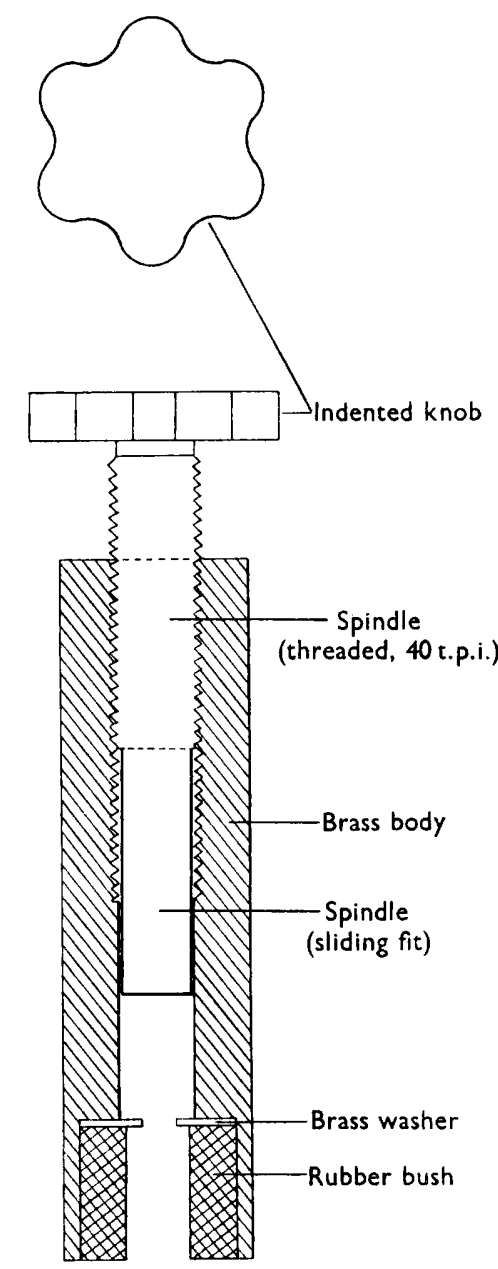

Fig. 1. L.S. pipette metering device $(\times 2)$.

\section{EXPLANATION OF PLATE}

Fig. 1. Reduction by 6.66 diameters of actual size of projected image.

(Received 19 December 1955) 